\title{
La gobernanza como principio de gobierno y convivencia social
}

\section{Governance as a principle of government and social coexistence}

\author{
Miguel Guerrero Olvera \\ ORCID: 0000-0003-0206-1117/mguerreroolvera 2002@yahoo.com.mx \\ Profesor-investigador, Facultad de Estudios Superiores de Cuautla (FESC), Universidad Autónoma del Estado de Morelos (UAEM)
}

\section{RESUMEN}

Se exponen las razones por las cuales se debe considerar adquirir la gobernanza como herramienta de gobierno para la integración social de los sujetos y una convivencia con los demás. Se explica cada uno de los elementos de este principio, que son el mecado, el Estado y la sociedad. Los dos primeros elementos tienen una estructura de poder; el mercado se rige por las leyes del capitalismo, y el Estado se rige por la legitimidad. Lo que busca la gobernanza es una nueva forma de gobierno que se base en la cooperación en vez de las jerarquias de poder, atender la complejidad de la realidad social y evitar los procesos de fragmentación que se manifiestan como consecuencia de las dinámicas individualistas.

\section{PALABRAS CLAVE}

gobernanza, principios de gobierno, mercado,

Estado, sociedad
ABSTRACT

This article explains the reasons why acquiring governance as a government tool for the social integration of the individuals and a coexistence with others must be considered. Each of the elements of this principle is explained: the market, the state and society. The first two elements have a power structure; the market is governed by the laws of capitalism, and the state is governed by legitimacy. What governance seeks is a new form of government based on cooperation instead of hierarchies of power, dealing with the complexity of social reality and avoiding the fragmentation processes that manifest as a consequence of individualistic dynamics.

\section{KEY WORDS}

governance, government principles,

market, state, society 
En el presente trabajo se reflexiona sobre el concepto de la gobernanza como principio de gobierno e integración social, que permite a los sujetos encontrar su lugar en la convivencia con sus semejantes. Se parte de la pregunta sobre si el mercado, el Estado y la sociedad, en donde prevalecen los principios de mando, competencia y lealdad, respectivamente, resultan ser, por separado, suficientes para tal fin, o, en caso contrario, si es necesario pensar en la gobernanza, en donde, de manera incluyente, se presentan estos principios para una conducción exitosa como práctica de gobierno.

Si por "gobierno" se entiende la acción de conducir el timón para llevar la nave a buen puerto, entonces el orden y la integración social de los gobernados es una condición de éxito de todo gobierno, por lo que además surge la pregunta sobre qué nave con la tripulación en motín o conflicto puede navegar exitosamente, bien sea el conflicto por los gobernados en competencia, o entre éstos y el gobernante por excesos de mando, o por lealtad y simpatías de los gobernados (Aznar y De Luca, 2010).

El fenómeno de la competencia ha derivado en tendencias individualistas en los sujetos que participan en el mercado; el exceso de mando ha dado lugar a Estados autoritarios, y la pasividad de los gobernados a gobiernos paternalistas (Vargas, 2006). Por lo anterior, la insuficiencia de los principios de competencia, mando y lealtad para ser rectores de la acción de gobierno y de procesos de integración social ha derivado en fallos de gobierno y en la necesidad de experimentar nuevas estrategias para conducir el timón. Fue así como surgió la gobernanza, cuya esencia está sustentada en la participación conjunta de actores provenientes del mercado, el Estado y la sociedad civil, y normados por principios no sólo de mando, competencia o lealtad y simpatía, sino de todos ellos en nuevas modalidades, como lo hemos de ver más adelante.

Es en este contexto de gobernanza donde adquiere sentido lo dicho por Catlin (2019) cuando definió la política como "interesada en las relaciones de los hombres, en asociación y competencia, sumisión y control, en la medida en que procuran [...] encontrar su lugar en la convivencia con sus semejantes" (p. 35), lo que hace permanente la búsqueda de esta necesidad en donde los diversos actores encuentran ese lugar que posibilite la convivencia con sus semejantes; la gobernanza, entonces, se presenta como una realidad asequible y necesaria.

\section{Mercado, Estado o sociedad: la antesala del conflicto}

\section{El mercado}

Los objetivos a alcanzar en el capitalismo son la acumulación, la productividad y la competitividad (Heilbroner, 1989), lo que provoca que a los individuos dentro de este sistema se les exija un comportamiento racional, es decir, un comportamiento de medios-fines que logra 
que sus actos sean calculables y calculadores de la ganancia a obtener, por lo que este comportamiento resulta ser eficiente y competitivo. La exigencia de este comportamiento deriva de la supuesta presencia de leyes objetivas, intrínsecas y anónimas, que condicionarían el comportamiento de los individuos para que se encauce a la búsqueda del máximo beneficio al menor costo posible, así como de una "mano invisible" que impulse la autorregulación del mercado y propicie un orden social como condición natural del capitalismo:

La economía de mercado supone un sistema autorregulador de mercados. Para emplear términos un poco más técnicos, se trata de una economía gobernada por los precios del mercado y únicamente por ellos. Sólo en este sentido se puede decir que un sistema de este tipo, capaz de organizar la totalidad de la vida económica sin ayuda o intervención exterior, es autorregulador (Polanyi, 2008, p. 85).

Mientras que la eficiencia se manifiesta en el proceso productivo, la competencia se presenta en el mercado como arena de confrontación de los intereses de los participantes, convirtiéndose unos y otros en contrincantes permanentes que buscarán su beneficio a partir de un comportamiento competitivo encaminado a imponerse a los intereses de los otros y con esto obtener el triunfo sobre ellos, propiciando su exclusión del mercado.

La competencia es una condición de sobrevivencia para quienes participan en el mercado, y obliga a los empresarios y comerciantes a confrontarse entre sí por la obtención de mercados, promoción y venta de sus productos, por lo que, a decir de Castoriadis (2008): "la competencia obliga al capitalismo, bajo pena de muerte, a perseguir el máximo resultado con el mínimo de medios" (p. 10). Esta condición de sobrevivencia también aplica para los empresarios en su relación con los trabajadores para lograr su máxima expoliación en forma de plusvalía, por lo que "[se] divide el tiempo de trabajo en 'tiempo de trabajo retribuido' (o trabajo necesario) y 'tiempo de trabajo no retribuido' (o trabajo excedente), siendo la plusvalía el tiempo de trabajo no retribuido que el asalariado deja en manos del capitalista" (Santarcángelo y Borroni, 2012, p. 4). Finalmente, también condiciona la relación entre los mismos trabajadores, quienes luchan entre ellos para acceder a un salario más retributivo, e incluso para acceder a las fuentes de trabajo, y así el trabajador "se halla en feroz competencia con centenares de colegas que se encuentran en la misma posición y si llega a dejarse superar es inexorablemente despedido" (Fromm, 2006, p. 158).

Por estas razones, en el capitalismo todo comportamiento de los diversos actores debe manifestarse en la búsqueda de los instrumentos y las maneras de operación que den lugar a la obtención de la mayor ganancia posible, lo que ha hecho que la competencia se convierta en la razón de ser de todo comportamiento en el mercado; de ahí que Castoriadis (2008) nos diga:

Año 17, núm. 41, marzo 2021

ISSN: 2007-1760 (impreso) 2448-9026 (digital) | DOI: 10.30973/inventio/2021.17.41/2 
La competencia obliga al capitalismo, bajo pena de muerte, a perseguir el máximo resultado con el mínimo de medios; ¿y acaso no es ése el fin mismo de la economía, la definición de su racionalidad? Y para lograrlo en grado creciente a la ciencia al servicio de la producción, racionaliza el proceso de trabajo por medio de esas encarnaciones de la razón operante que son ingenieros y técnicos (p. 10).

Sin embargo, hoy día esta racionalidad y el mercado que la encarna se manifiesta en crisis permanente como consecuencia de las contradicciones del capitalismo, que, a decir de Abdalla (2009), ha dado lugar a que:

Entre los seres humanos, los efectos de esa racionalidad crean un clima de tensión constante. [...] De esa tensión resultan los innumerables conflictos sociales existentes. Esos conflictos suceden en el interior mismo de las clases sociales, lo que explica en parte tanto el descontrol del mercado financiero, la quiebra de competidores, etc., como la violencia que se propaga entre las clases subordinadas de diversos países (sección 3, párr. 20).

Por lo hasta aquí señalado, bien podemos afirmar que la competencia, en tanto principio regulador del comportamiento de los sujetos, lejos se encuentra de propiciar un punto de encuentro para la convivencia entre ellos. Veamos otras opciones.

\section{El Estado}

De acuerdo con Passerin (2001), los principios rectores de la acción del Estado para impulsar procesos de integración social a través de la acción de gobierno son la fuerza, el poder y la autoridad. En su origen, se manifestó la fuerza, que proporciona una idea ambigua de la voluntad individual y superior a ella, hasta el punto de no dar sólo mandatos, sino de exigir su ejecución. Sin embargo, con el paso del tiempo, la acción de gobierno demandó algo más que el ejercicio de la fuerza: exigió la presencia del poder; de un poder que pasó a identificarse con el ejercicio de la ley, y modificó el comportamiento del Estado, que pasó del ejercicio arbitrario de la dominación por el monarca a una acción cuyo rasgo será la impersonalidad de la norma. Finalmente, en esta evolución del Estado surgió otro principio regulador de su comportamiento: la legitimidad. Lo anterior ocasionó que el Estado no solamente se ocupara de cómo ejercer el poder, sino también de dar cuenta del porqué y para qué de este ejercicio.

Lo hasta aquí señalado nos permite afirmar que, al Estado, para el cumplimiento de su función de gobierno y para manifestar su fortaleza como centro de poder, le resulta necesario ser legitimado a partir del consenso de los individuos, lo que habrá de fortalecer su presencia por el grado de aceptación de sus mandatos. 
El consenso bien puede ser otorgado al Estado por medio de la legitimidad que Max Weber (2012) denominó tradicional, carismática o legal-racional. En el caso de la primera, se corresponde con el consenso otorgado a la acción de gobierno como consecuencia de ser permanente y constante, es decir, identificada con el eterno ayer, con el "siempre ha sido así". En la legitimidad carismática el consenso o la aceptación de los actos de gobierno van a derivar de las cualidades intrínsecas que posee quien las emite. Mientras que la legitimidad legal-racional se va a caracterizar por la exigencia de que los actos de quien gobierna se correspondan con lo que la ley señala, es decir, este tipo de legitimidad implica la simbiosis entre legalidad y legitimidad.

Hemos de señalar que este último tipo de legitimidad con el paso del tiempo resultó insuficiente, dadas las condiciones de vida que han venido enfrentado los diversos grupos sociales mayoritarios, e indujo a que las acciones de gobierno atendieran la merma en sus condiciones de vida. Lo anterior dio lugar al surgimiento del Ilamado Estado de bienestar, que consiste en buscar la aceptación de los individuos a través de un intercambio material que satisfaga sus necesidades, pero éstos deben aceptar la toma de decisiones del gobierno. A esto, Gianfranco Poggi (1997) lo nombró eudemonía social, esto es, un tipo de legitimidad derivada de grado de satisfacción dada por el Estado a los requerimientos materiales y de bienestar de los individuos.

Por tal motivo, entre las diversas acciones acometidas por el Estado para el cumplimiento de esta función de bienestar social, y para materializar su fortaleza como centro de poder y autoridad, encontramos su capacidad de control de los instrumentos coercitivos e impositivos de la observancia de las normas y los procedimientos legales, así como en el control de las decisiones respecto de la distribución de recursos, la organización de los servicios colectivos y la gestión del conjunto de la sociedad.

Sin embargo, esta forma de proceder del Estado ha recibido variadas y permanentes críticas por el empleo de la racionalidad instrumental que la caracteriza al erigirse como instancia única de regulación social bajo la pretensión de poseer la capacidad de determinar el proceder de la sociedad y de encaminarla hacia fines determinados por su ejército de expertos, así como por el tipo de intercambio perverso de satisfacción a las demandas de la sociedad por lealtad al régimen, y ha derivado en gobiernos clientelistas y populistas, los cuales también son objeto de fuertes cuestionamientos por impedir a los individuos, a través del Estado, encontrar su lugar en la convivencia con sus semejantes. Veamos ahora cómo la sociedad coadyuva o no para tal fin.

\section{La sociedad}

Los individuos, cuando participan en un colectivo, el principio que va a regular su comportamiento es la cooperación. Esto se observa desde el origen de la sociedad, cuando la condición para vincularse con otros individuos fue la adversidad que se enfrentó para lograr la 
sobrevivencia. Un principio fundamental que se manifestó al interior de los grupos fue la división del trabajo, que los indujo a generar formas colectivas del desempeño sustentadas en la cooperación, por ser indispensable para hacerse de los bienes necesarios para su sobrevivencia.

La cooperación, entonces, se manifestó como una condición para la existencia de todo grupo social, dado el establecimiento de formas de intercambio en las que los individuos encontrarían formas de beneficio compartido, por lo que "no existe ni es imaginable siquiera un Yo sin un Tú, un Ellos, un Nosotros. Es imposible la construcción aislada de una identidad individual, ya que el individuo logra tomar conciencia de su individualidad solamente por medio de la mirada del Otro" (Lechner, 2000, sp).

Cabe señalar que la cooperación lejos se encuentra de aquella postura en donde se considera al otro como el contrario, como rival al que se debe vencer; contrariamente, en la cooperación el otro se convierte en un complemento, pero no como una imposición, sino como resultado de una relación dialéctica sustentada en la necesidad de unificar criterios diversos para lograr una posición compartida que permita atender los enormes problemas de fragmentación que ha generado el capitalismo por convertir al mercado en el espacio donde el fin último es la competencia.

Por otro lado, no podemos pensar que la cooperación se produce de manera espontánea, sino que surge de un proceso intenso de formación y educación que permite a los individuos asimilar una nueva racionalidad para aprender a estar juntos, a convivir con los otros. Una convivencia en la que, pese a las diferencias, sean capaces de llegar a acuerdos comunes que permitan la sobrevivencia. En ello será importante la presencia del diálogo como condición indispensable.

Esta necesidad de diálogo ha sido nombrada por Richard Sennett (2012) como una habilidad dialógica que "designa la atención y la sensibilidad en relación con otras personas, [...] es el arte de escuchar, de proyectarse hacia el exterior, [por lo] que reduce el ego" (p. 31). Mediante la habilidad dialógica la cooperación se construye de abajo hacia arriba, ajena a todo tipo de imposición que la convierta en sólo un fin y no un medio para objetivos distantes del beneficio de los participantes. Mediante esta habilidad surge la empatía como condición indispensable para que el encuentro no derive en una práctica simplemente emotiva, irreflexiva, que ponga en riesgo el beneficio común. La empatía coloca la atención de forma íntegra en el otro y no sólo en sus afectos; la atención se centra no en lo que siente, sino en lo que expresa de forma dialógica.

Las bondades que puede representar una integración social sustentada en la cooperación, y que los individuos encuentren su lugar en la convivencia con sus semejantes mediante la empatía, existe siempre latente. Sin embargo, el riesgo de que las relaciones se sustenten más en la simpatía que en la empatía da lugar a formas de relación paternalistas en las 
que uno de los sujetos pasa a convertirse en un director, un guía, un sujeto poco dispuesto y proclive a la cooperación y al reconocimiento del otro como un sujeto activo digno de plena confianza, por lo que reproduce modelos de integración social autoritarios que convierten al otro, u otros sujetos, en piezas leales y pasivas de esta relación paternalista y autoritaria.

\section{La gobernanza a escena}

Fue importante realizar lo siguiente ante las decadencias por impulsar las formas de integración social que permiten a los individuos encontrar un lugar en la convivencia con sus semejantes, que pueden provenir del mando y de la competencia o de la lealtad y simpatía:

Un nuevo estilo de gobierno, distinto del modelo de control jerárquico y caracterizado por un mayor grado de cooperación y por la interacción entre el Estado y los actores no estatales al interior de redes decisionales mixtas entre lo público y lo privado (Prats, 2005).

Por tal motivo, la gobernanza deberá ser producto de una interacción exitosa entre los diversos actores participantes, y sus valores han de ser la equidad, la solidaridad, la justicia y la corresponsabilidad, pero fundamentalmente la tolerancia. Esta última es un valor indispensable para la acción colaborativa de manera incluyente, lejana de la imposición y de la disciplina (que caracterizan la acción política del Estado); de la competencia, que termina siendo excluyente, y de la simpatía social, que da lugar a formas de relación paternalistas que represen la acción política del Estado. Derivado de lo anterior, Luis Aguilar explica que:

La Gobernanza incluye, por consiguiente, dos planos de decisión, en cada uno de los cuales tienen lugar interlocuciones, ajustes y acuerdos entre los actores participantes. Por un lado se decide sobre las preferencias, fines, objetivos y metas de valor para la sociedad en su conjunto o para específicos sectores sociales $y$, a la luz de esas referencias valorativas, se deciden las reglas de relación entre actores — las instituciones_ que deberán seguirse para ordenar las interacciones de la vida social (Aguilar, 2009, pp. 29-30).

\footnotetext{
${ }^{1}$ El tratamiento que se hace aquí de la gobernanza no pretende ser limitativo, pero se corresponde con los fines del presente documento, sin por ello dejar de lado lo que, a decir de Francisco Porras, nos señala Renate Mayntz: "la teoría de la gobernanza (en singular) ha sufrido extensiones consecutivas, obligándose a incorporar nuevos elementos conceptuales y referentes empíricos. Así, la primera extensión incluiría la perspectiva de abajo hacia arriba (bottom-up), incorporando las visiones y comportamientos de los destinatarios inmediatos de la política pública. La segunda extensión añadiría la perspectiva de las redes en el diseño e implementación de las políticas públicas; la tercera evidenciaría el efecto de las decisiones de la Unión Europea sobre las políticas públicas nacionales; la cuarta sumaría el nivel europeo como un verdadero nivel de gobierno supranacional, y la quinta reconocería los procesos políticos, incluyendo las propuestas" (Mayntz, 2005, citado en Porras, 2016, p. 163).
}

Año 17, núm. 41, marzo 2021

ISSN: 2007-1760 (impreso) 2448-9026 (digital) | DOI: 10.30973/inventio/2021.17.41/2 
En la gobernanza cabría esperar un comportamiento distinto de los actores provenientes de los diversos sectores: el mercado, el Estado y la sociedad. En el caso de los actores provenientes del mercado, debería darse un tipo de competencia nombrada como "todos ganan", es decir, aquélla donde la participación de los particulares bajo una lógica de mercado ya no podrá ser depredadora, sino un tipo de competencia en la que todos los que participan reciben algo, y ese algo habrá de ser producto de la negociación y del establecimiento de reglas claras que logran el proceso de interacción entre los diversos actores.

La participación del Estado debería darse como una participación distante de su afán impositivo mediante leyes y reglamentos, en donde las leyes prohíben y los reglamentos indican cuál ha de ser la forma de proceder de los individuos. Debería permitirse un comportamiento de los actores estatales en el que, lejos de la ley y de la regla, prevalezca un sentido de regulación de los procesos establecidos y formulados por los actores sociales, y que esta regulación manifieste un reconocimiento del interés común y el conocimiento que estos últimos actores tienen de su realidad, y no de la imposición de pretendidos expertos a través de normas y reglas eternas.

Los actores que provienen del terreno social deberían manifestar un comportamiento encaminado hacia la cohesión social y, en consecuencia, a la reducción de la fragmentación que exista al interior de los procesos sociales como consecuencia de las diferencias que caracterizan a los sujetos; diferencias que habrán de ser superadas por prácticas permanentes de comunicación y diálogo que logren establecer criterios de atención diferenciada, pero equitativa, de los diversos intereses presentes.

Por tal motivo, la participación social tendrá que darse en pleno reconocimiento de que la ciudadanía no es algo dado, ni por el Estado, ni por ninguna ley o regla, es decir, la ciudadanía no se construye por decreto, sino con base a prácticas derivadas del marco institucional existente. El marco institucional cae en el terreno de la responsabilidad del Estado para llevar a cabo su regulación, pero no su formulación, ya que este marco deberá ser producto de los acuerdos y consensos establecidos por los participantes en el terreno social, de lo que derivará en la necesidad de un consenso logrado a través de los individuos y construido mediante el acuerdo y la discusión colectiva a través de los argumentos presentados.

Nos acercamos al tema de la democracia como un elemento indispensable en la conformación de estos acuerdos, gracias a que derivan de la participación y el diálogo pero no de una democracia formal sólo identificada con la emisión del voto, sino resultado de procesos colectivos, autónomos, abiertos y de deliberación, en los que la igualdad, el pluralismo y la solidaridad permitan superar las diferencias derivadas de los intereses de cada uno de los actores, de sus valores, opiniones y creencias, que no por ser diferenciados deben dar lugar al conflicto, sino que exigen reglas que, habiendo sido discutidas y argumentadas por los participantes, dan lugar a consensos para establecer mecanismos de interacción, cohesión y convivencia social. 
Lo hasta aquí dicho nos permite vislumbrar la gobernanza no como un campo idílico de buenas intenciones o cumplimiento de valores universales, sino de prácticas de negociación y conciliación que no nieguen el conflicto, pero sí que les permitan a los diversos actores transitar hacia la construcción de acuerdos y encuentros que resultan necesarios hoy en día para la atención de la complejidad de los diversos procesos sociales y que posibiliten la presencia de la cohesión social como una condición indispensable para la supervivencia de todo grupo social, para que los individuos encuentren su lugar en la convivencia con sus semejantes, como fue referido por Catlin (2019) cuando definió la política.

\section{Conclusiones}

La gobernanza es una condición indispensable para atender la complejidad de la realidad social y para evitar los procesos de fragmentación que se manifiestan como consecuencia de las dinámicas individualistas y de confrontación de intereses que generalmente caracterizan a toda sociedad, por lo que deberá ser resultado de procesos de participación colectiva en donde la cooperación desempeñe un papel preponderante.

Con este tipo de participación, en busca del mayor grado posible de cohesión social, se pretende superar la racionalidad y las contradicciones que son producto de procesos económicos generadores de individualismos a ultranza y de procesos de fragmentación social como consecuencia de la polarización de intereses por la presencia de mecanismos distributivos inequitativos de la riqueza. También se pretende superar la lógica de la participación de actores gubernamentales manifestada en formas de gobierno autoritarias y de procesos deterministas de planificación de la realidad, sustituyéndolos por procesos democráticos en los que se manifieste la corresponsabilidad, la igualdad, el pluralismo, la deliberación y la solidaridad, en tanto nuevo modelo de gobierno por gobernanza, en el que se hagan presentes procesos de cooperación que sustituyan a los procesos tradicionales de autoridad derivados del protagonismo estatal y de la competencia del tipo "suma cero" proveniente del mercado.

Es en este sentido que la gobernanza implica la presencia de procesos de cohesión encaminados a la superación de los procesos de fragmentación social resultantes de criterios mercantiles, autoritarios o paternalistas, para dar lugar a un mayor grado de gobernabilidad que derive de que los encuentros entre individuos con intereses diferenciados se hagan posibles a través de la negociación y la conciliación de intereses, en pro de la supervivencia de la sociedad, y de procesos permanentes de autoconservación de ella. 


\section{Referencias}

Abdalla, M. (7 de abril de 2009). El principio de cooperación. En busca de una nueva racionalidad. Evolución y Ambiente. https://www.iieh.com/index.php/categories/ evolucion-ambiente/de-fondo/el-principio-de-cooperacion

Aguilar, L. (2009). Gobernanza: normalización conceptual y nuevas cuestiones. CuadernoS de Liderazgo, 17. ESADE.

Aznar, L. y De Luca, M. (2010). Política. Cuestiones y problemas. Cengage Learning.

Castoriadis, C. (2008). La lucha de los obreros contra la organización de la empresa capitalista. El pensamiento de Cornelius Castoriadis (vol. 1). Ediciones Proyecto Revolucionario.

Fromm, E. (2006) Psicoanálisis de la sociedad contemporánea. Hacia una sociedad sana. FCE.

Catlin, G. E. G. (2019). The Science and method of politics. Routledge Library Editions.

Heilbroner, R. L. (1989). Naturaleza y lógica del capitalismo. Siglo XXI.

Lechner, N. (2000). Desafíos de un desarrollo humano: individualización y capital social. Instituciones y Desarrollo, 7, 7-34.

Passerin, A. (2001). La noción de Estado. Una introducción a la teoría política. Ariel.

Poggi, G. (1997). El desarrollo del Estado moderno. Una introducción sociológica. UNQ.

Polanyi, K. (2008). La gran transformación. Quipu editorial.

Porras, F. (2016). Gobernanza. Propuesta, límites y perspectivas. Instituto Mora.

Prats, J. (2005). De la burocracia al management, del management a la gobernanza. Las transformaciones de las administraciones públicas de nuestro tiempo. INAP.

Santarcángelo, J.E.y Borroni, C. (2012). El concepto de excedente en la teoría marxista: debates, rupturas y perspectivas. Cuadernos de Economía, 31 (56), 1-20. https://www.redalyc.org/ pdf/2821/282124593001.pdf

Sennett, R. (2012). Juntos. Rituales, placeres y políticas de cooperación. Anagrama.

Vargas, J. G. (2006). La gobernabilidad del Estado, el mercado y la sociedad. El Cotidiano, 21 (137), 82-91. https://www.researchgate.net/publication/26483261 La Gobernabilidad del Estado el mercado y la sociedad

Weber, M. (2012). Economía y sociedad. FCE. 